\title{
A case of Poncet's disease; a mimicker of rheumatoid arthritis
}

\author{
Dharmaratne $\mathbf{C J}^{1}$, Jayasena DP ${ }^{1}$, Sobana VM ${ }^{1}$, De Silva UD ${ }^{1}$, Muhunthan $\mathrm{S}^{2}$ \\ Department of Clinical Medicine, Teaching Hospital Karapitiya, Galle, Sri Lanka. \\ Department of Respiratory Medicine, Teaching Hospital Karapitiya, Galle, Sri Lanka.
}

\author{
Co-Correspondence: Dr. C.J. Dharmaratne; e-mail: charukad@gmail.com \\ D https://orcid.org/0000-0002-2370-1606 \\ Dr.D.P. Jayasena; e-mail: pravja1988@gmail.com \\ (D) https://orcid.org/0000-0002-9966-3773 \\ Submitted on 04.07.2021 and accepted for publication on 29.07.2021
}

\section{Introduction}

Tuberculosis (TB) has a variable clinical presentation affecting the lungs, lymph nodes, pleura, gastrointestinal tract, meninges, joints, and bones. Musculoskeletal involvement is the most common manifestation of extrapulmonary TB, seen in $10-19 \%$ of cases (1). Although septic TB arthritis is a well-recognized manifestation of extrapulmonary TB; there is another rare form of reactive arthritis known as Poncet's disease (PD). $\mathrm{PD}$ is an aseptic non-erosive reactive arthritis that occurs with active extra-articular TB without any long term complications (2). It was first described by Antonin Poncet in 1897 as a polyarthritis secondary to visceral TB (3). The pathogenesis of PD remains unknown, however, it is strongly suspected to be due to Mycobacterium tuberculosis inducing a $\mathrm{T}$ helper 1 cell-mediated cross-reaction between tuberculoprotein and cartilage proteoglycans which trigger inflammatory arthritis (3). Therefore, antigen molecular mimicry and cross-reactivity have been implicated in the pathogenesis of $\mathrm{PD}$ in genetically predisposed individuals with HLA DR 3 and HLA DR 4 haplotypes (3-5). PD typically presents as a symmetrical large and small joint polyarthritis during an active TB infection with aseptic joints mimicking inflammatory arthritis (3). The focus of TB in majority of cases was TB lymphadenitis $(3,4)$.

There are no specific investigations to diagnose PD, hence it is diagnosed by clinical assessment and exclusion of other probable causes of inflammatory arthritis $(3,4) . P D$ is a frequently misdiagnosed entity even in high TB prevalent countries due its unusual and uncommon presentation (6). Misdiagnosis and treatment as inflammatory arthritis with immunosuppressive medication will lead to dissemination of TB (7). Commencement of anti-TB treatment completely resolves symptoms within weeks to months and furthermore, confirms the diagnosis of PD $(1,3,4)$. To the authors' knowledge, this is the first reported case of PD in Sri Lanka.

\section{Case presentation}

A 66-year-old male street vendor presented with low-grade evening pyrexia, fatigue, night sweats, loss of appetite and weight for 4 months. He had active arthritis effecting bilateral proximal interphalangeal joints (PIPs), metacarpophalangeal joints (MCPs), wrist joints (WJs), elbow joints (EJs), knee joints (KJs), ankle joints (AJs) and metatarsophalangeal (MTPs) joints for 3 months despite treatment with analgesics and had affected most of his activities of daily living. The pain worsened with activity but no associated morning stiffness. There was no history of diarrhoea, eye symptoms, skin rashes and genitourinary symptoms or personal/ family history of tuberculosis or any other comorbidities. He had a history of smoking, approximately 5 packets of cigarettes per year and did not consume alcohol.

His body mass index (BMI) was $16 \mathrm{~kg} / \mathrm{m}^{2}$. He was pale, afebrile, and anicteric. There was no palpable 
lymphadenopathy. His cardiovascular, respiratory, abdominal, and nervous system examinations were normal. Musculoskeletal system examination revealed active synovitis and limited range of movements in the above-mentioned joints. Full blood count (FBC) showed anaemia of chronic disease $(\mathrm{Hb}-9 \mathrm{~g} / \mathrm{dl})$ with normal WBC $\left(7.3 \times 10^{9} / \mathrm{L}\right)$ and neutrophil count $\left(4.2 \times 10^{9} / \mathrm{L}\right)$ with thrombocytosis (platelet - $572 \times 10^{9} / \mathrm{L}$ ). The ESR was $130 \mathrm{~mm} / \mathrm{hr}$ and CRP was $20 \mathrm{mg} / \mathrm{dl}$.

$\mathrm{He}$ had markedly elevated rheumatoid factor (RF) of $380 \mathrm{IU} / \mathrm{ml}$ and anti-cyclic citrullinated peptide (anti-CCP) titre was $110 \mathrm{IU} / \mathrm{ml}$. Moreover, antinuclear antibodies (ANA), antineutrophil cytoplasmic antibodies (ANCA), anti-streptolysin $\mathrm{O}$ titre (ASOT), serum calcium, bone profile markers and HIV antibodies were negative. Right $\mathrm{KJ}$ synovial fluid analysis showed leucocytes $\left(8 \times 10^{9} /\right.$ L) with polymorph predominance and no crystals. The synovial fluid gram stain, bacterial and TB cultures were negative. Synovial biopsy revealed mild chronic inflammatory cell infiltrate with no histological evidence ofTB.

Radiographs of hands, wrists, knees, and ankle joints did not reveal any erosive changes and chest X-ray revealed left upper lobe cavitory lesions with mild volume loss (Figure 1). Three sputum samples were negative for acid-fast bacilli. However, he had a strongly positive Mantoux test of $16 \mathrm{~mm}$ induration. His liver function, renal function and serum electrolytes were normal.

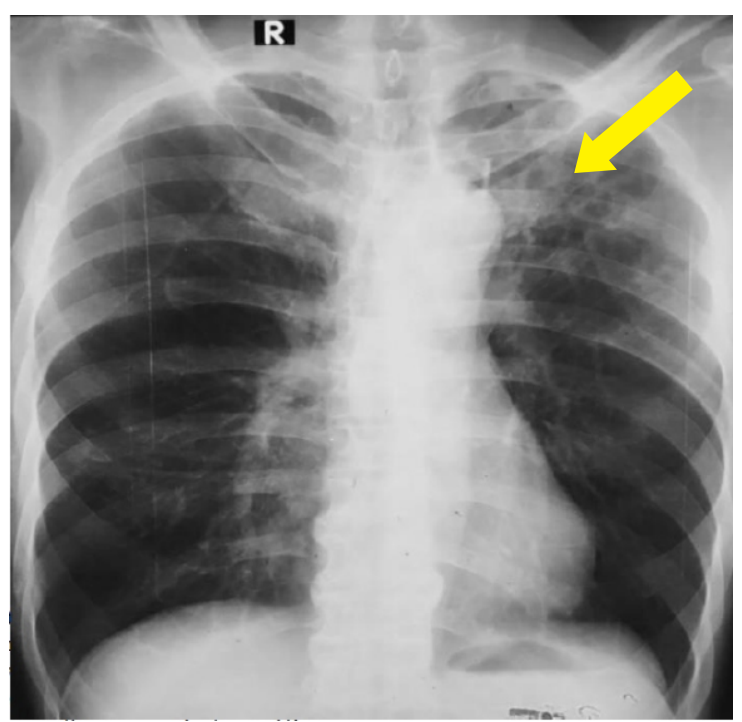

Figure 1: Left apical cavitation demonstrated on Chest X-ray (arrow)
The high-resolution computed tomography chest showed thin-walled cavities with fibrosis and volume loss in the left apico-posterior segment suggestive of post-pulmonary tuberculosis. Following case discussion with the pulmonologist, we proceeded with bronchoscopy where the bronchoalveolar lavage (BAL) for TB polymerase chain reaction (PCR) revealed a moderate yield of TB DNA and BAL culture subsequently grew Mycobacterium tuberculosis.

After multidisciplinary team discussion including general physician, respiratory physician and Rheumatologist, patient was commenced on the category 1 anti-TB treatment (isoniazid $5 \mathrm{mg} / \mathrm{kg}$, rifampicin $10 \mathrm{mg} / \mathrm{kg}$, pyrazinamide $25 \mathrm{mg} / \mathrm{kg}$ and ethambutol $15 \mathrm{mg} / \mathrm{kg}$ ) according to the World Health Organisation (WHO) tuberculosis guidelines (8). At three months his joint pains have completely resolved. He was continued on anti-TB treatment for 6 months in total and fully recovered.

\section{Discussion}

PD is a very rare entity, where no more than 200 cases published in the literature (7). So far there have been no cases of PD reported in Sri Lanka, to the authors' knowledge. Whilst symmetrical aseptic polyarthritis involving large joints remain the classic presentation of PD, the majority of cases were reported to be asymmetrical non-destructive oligoarthritis with predominant ankle involvement (3). Joint symptoms could last from three days to six years (3). Associated constitutional symptoms were fever, malaise and weight loss $(1,3)$. Moreover, cervical and axillary lymphadenopathy along with erythema nodosum were observed in up to $6 \%$ of patients with PD.

Despite RF and anti-CCP antibodies being useful markers in the diagnosis of rheumatoid arthritis, they were found to be positive in patients with mixed cryoglobulinaemia, TB, infective endocarditis, primary biliary cirrhosis and malignancy $(1,9)$. Tuberculosis infections in particular were associated with seropositive RF (8-60\%) and anti-CCP (7-39\%) antibodies $(1,9)$. Furthermore, seropositivity of RF and anti-CCP antibodies have been reported in PD similar to our patient fulfilling American College of Rheumatology and European League Against 
Rheumatism criteria for Rheumatoid arthritis $(1,8,9)$. Therefore, RF and anti-CCP seropositive arthritis should not automate to the diagnosis of rheumatoid arthritis $(1,2,8,9)$. There are no standard definitions and diagnostic criteria widely used in clinical practice to identify PD $(3,4)$. Thus diagnosing PD is made by exclusion of other causes of inflammatory polyarthritis $(3,4,9)$. However there is a proposed diagnostic criterion by Sharma and Pinto (Table 1) who applied it in their case series, yet it needs to be validated in prospective cohort studies and different population groups (3).

Despite the elevated inflammatory markers, the aseptic nature of his involved joints was confirmed by the sterile synovial fluid cultures and absent caseating granulomatous necrosis in the synovial biopsy, thus making TB arthritis an unlikely diagnosis. PD arthritis occurs concomitantly with the onset of infection accompanied by extra-articular manifestations whereas reactive arthritis occurs 1 - 4 weeks after the infection (1-3,9). Unlike reactive arthritis, PD is a non-erosive arthritis sparring axial and sacroiliac joints (3). PD significantly improved with anti-TB treatments over several weeks in contrast to reactive arthritis which requires nonsteroidal anti-inflammatory drugs, glucocorticoids or DMARDs (full term) with a slower recovery over 6 months $(1,2,9)$.
Although it is possible to consider the co-existence of pulmonary TB and rheumatoid arthritis there was clear evidence of rapid remission of polyarthritis and dramatic decline of inflammatory markers following anti-TB treatment. Therefore this outcome favoured PD, unlike rheumatoid arthritis where his polyarthritis would have persisted despite antiTB therapy $(1,9)$. Further, according to Sharma and Pinto's diagnostic criteria, our patient is a definite case of $\mathrm{PD}(3)$.

\section{Conclusions}

$\mathrm{PD}$ is a rare form of aseptic arthritis in the presence of pulmonary or extrapulmonary TB which mimics classic inflammatory arthritides such as rheumatoid arthritis and there are no validated diagnostic criteria that make it challenging to diagnose $(1,3,9)$. This case study emphasizes the importance of including $\mathrm{PD}$ as a differential diagnosis in patients presenting with fever and polyarthritis or oligoarthritis especially in countries with high TB prevalence $(4,9)$.

Table 1: Sharma and Pinto's diagnostic criteria for Poncet's disease

$$
\begin{array}{ll}
\hline \text { Essential criteria } & \text { Inflammatory, non-erosive, non-deforming arthritis } \\
& \text { Exclusion of other causes of inflammatory arthritis }
\end{array}
$$

$\begin{array}{ll}\text { Major criteria } & \begin{array}{l}\text { Concurrent diagnosis of extra-articular tuberculosis } \\ \text { Complete response to antitubercular therapy }\end{array}\end{array}$

Minor criteria $\begin{aligned} & \text { 1. Mantoux positivity } \\ & \text { 2. Associated hypersensitivity phenomenon, } \\ & \text { such as erythema nodosum, tuberculids or phlyctenular } \\ & \text { keratoconjunctivitis } \\ & \text { Absence of sacroiliac and axial involvement }\end{aligned}$
For diagnosis:
Definite - Essential + two major
Probable - Essential + one major + three minor
Possible - Essential + one major + two minor, or
$\quad-$ Essential + three minor




\section{References}

1. Sasaki H, Inagaki M, Shioda M, Nagasaka K. Poncet's disease with high titers of rheumatoid factor and anticitrullinated peptide antibodies mimicking rheumatoid arthritis. J Infect Chemother [Internet]. 2015;21(1): 65-9.

2. Kroot EJA, Hazes JMW, Colin EM, Dolhain RJEM. Poncet's disease: Reactive arthritis accompanying tuberculosis. Two case reports and a review of the literature. Rheumatology. 2007; 46(3): 484-9.

3. Sharma A, Pinto B, Dogra S, Sharma K, Goyal P, Sagar V, et al. A case series and review of Poncet's disease, and the utility of current diagnostic criteria. Int $J$ Rheum Dis [Internet]. 2016 Oct 1; 19(10): 1010-7.

4. Chakraborty PP, Ray S, Selvan C, Bhattacharjee R, Mandal SK. Poncet's disease: An unusual presentation of tuberculosis in a diabetic lady. World J Clin cases [Internet]. 2015 Apr 16;3(4): 385-8.

5. Adhi F, Hasan R, Adhi M, Hamid SA, Iqbal N, Khan JA. Poncet's disease: two case reports. 2017; 1-5.
6. Mehrotra V, Garg K, Devi P, Chauhan S. A commonly missed diagnosis: Poncet's disease. I Indian Acad Oral Med Radiol. 2019;31(2): 176.

7. Es-Souiri J, Mounir A, Kona I, Abourazzak FE, Harzy T. Poncet's disease: A challenging cause of polyarthritis in a Moroccan patient. Egypt Rheumatol [Internet]. 2017; 39(3): 183-5.

8. Nyo MTL, Ally MMTM, Van Duuren EM, Arendse R. Poncet's Disease in the Preclinical Phase of Rheumatoid Arthritis. Hsu T-C, editor. Case Rep Rheumatol 2018; 2018: 3571247. doi: 10.1155/2018/3571247.

9. Ariza-Prota M, Pando-Sandoval A, García-Clemente M, Casan P. Poncet's disease mimicking rheumatoid arthritis in a patient with suspected Crohn's disease. Clin case reports. 2015 Nov 23; 4(1): 72-5. 